\title{
Role of the glucocorticoid receptor in the recurrence of primary nephrotic syndrome
}

\author{
YUMEI LIANG, YINYIN CHEN, YING CHEN, and YUTING GONG \\ Department of Nephrology, Hunan Provincial People's Hospital, First Affiliated Hospital of Hunan Normal University, \\ Changsha, Hunan 410005, P.R. China
}

Received November 1, 2013; Accepted July 16, 2014

DOI: $10.3892 /$ etm.2015.2665

\begin{abstract}
The present study aimed to investigate the changes in the expression levels of the glucocorticoid receptor (GR) and its subtypes in patients with recurrent renal syndrome. In addition, the effects of tumour necrosis factor $\alpha$ (TNF- $\alpha$ ) and a TNF- $\alpha$ monoclonal antibody on these receptors in peripheral blood mononuclear cells (PBMCs) isolated from the patients was analysed. Furthermore, a new treatment method for recurrent renal syndrome was explored. The serum levels of TNF- $\alpha$ in the normal (A), stable renal syndrome (B) and renal syndrome recurrence $(\mathrm{C})$ groups of patients were determined by enzyme-linked immunosorbent assay (ELISA). The mRNA and protein expression levels of GR, GR $\alpha$ and GR $\beta$ were determined by ELISA, western blot analysis and quantitative polymerase chain reaction in PBMC cultures from the three groups in the absence of intervention (blank control) and following stimulation with methylprednisolone, TNF- $\alpha$ and/or TNF- $\alpha$ monoclonal antibody. Group C exhibited higher expression levels of TNF- $\alpha$ and GR $\beta$ but a lower level of GR $\alpha$ expression $(\mathrm{P}<0.05)$ compared with the other groups. Regardless of methylprednisolone intervention, the expression levels of GR and GR $\beta$ in the three groups following stimulation by TNF- $\alpha$ were significantly higher compared with those in the respective blank control, whereas in group $\mathrm{C}$, the GR $\alpha$ expression levels following TNF- $\alpha$ treatment were lower compared with those in the control group $(\mathrm{P}<0.05)$. The treatment of group $\mathrm{C}$ with TNF- $\alpha$ monoclonal antibodies resulted in higher GR $\alpha$ expression but lower GR $\beta$ expression compared with those in the blank control $(\mathrm{P}<0.05)$. The change in the ratios of the GR subtypes may be associated with renal syndrome recurrence. TNF- $\alpha$ may be involved in renal syndrome relapse by changing the levels of GR as well as the
\end{abstract}

Correspondence to: Professor Yumei Liang, Department of Nephrology, Hunan Provincial People's Hospital, First Affiliated Hospital of Hunan Normal University, 61 Jiefang West Road, Changsha, Hunan 410005, P.R. China

E-mail: liang.yumei@aliyun.com

Key words: glucocorticoid receptor, nephrotic syndrome recurrence, tumor necrosis factor- $\alpha$, tumor necrosis factor- $\alpha$ monoclonal antibody proportion of the GR subtypes. TNF- $\alpha$ monoclonal antibodies may mitigate the changes in the ratios of these subtypes.

\section{Introduction}

Nephrotic syndrome is a common immune inflammatory kidney disease that accounts for $70-90 \%$ and $21-31 \%$ of glomerular diseases in children and adults, respectively (1). This syndrome is the leading cause of mortality in children with kidney disease in Asian countries (1). The efficiency of the treatment for nephrotic syndrome has improved in recent years. However, the recurrence of this disease, particularly the high recurrence rate caused by infections, remains a major clinical problem. No definitive conclusions on the mechanisms underlying nephrotic syndrome relapse have been reported. Several studies have revealed that nephrotic syndrome recurrences are associated with: the histological type of the nephridial tissue, renal renin-angiotensin gene polymorphisms, hyperthyroidism, expression of the tissue factor channel inhibitor, changes in human phospholipase A2 receptor expression, T-lymphocyte subsets, number and functional imbalance in proinflammatory cytokine secretion, peripheral blood mononuclear cell (PBMC) receptor affinities, hormone glucocorticoid (GC) pharmacokinetics and other factors (1-13). The association between the glucocorticoid receptor (GR) and the course and prognosis of renal syndrome has been extensively investigated in recent years (2-6). GCs, which are the first-choice drugs for renal syndrome treatment, exert their biological effects mainly through binding to GRs. GR $\alpha$ and GR $\beta$ are the two main subtypes of human GRs. Numerous studies have demonstrated that nephrotic syndrome recurrence is closely associated with the levels of GR, subtype percentage and activity changes (13-16). Inflammatory mediators and transcription factors, particularly the key inflammatory cytokine tumour necrosis factor $\alpha$ (TNF- $\alpha)$, are vital to the regulation of the efficacy of GR-mediated GC.

In the current study, the effect of TNF- $\alpha$ on GR mRNA and protein expression, as well as on the levels of the GR $\alpha$ and GR $\beta$ subtypes were investigated in vitro in peripheral blood mononuclear cells (PBMCs) collected from patients, and TNF- $\alpha$ monoclonal antibody intervention was used as a control. Serum TNF- $\alpha$ concentrations were also detected in patients. Changes in the levels of GR in patients with recurrent renal syndrome were determined and the function of the 
GR in renal syndrome recurrence was investigated. A possible mechanism underlying nephrotic syndrome recurrence was identified and alternative methods with improved GC reactivity and those involving TNF- $\alpha$ intervention were developed.

\section{Subjects and methods}

Subjects. A total of 25 inpatients who satisfied the domestic nephrotic syndrome (hereinafter referred to as renal syndrome) diagnostic criteria (1) were selected for participation in the present study. The patients were admitted to the Nephrology Department of the Second Xiangya Hospital of Central South University (Changsha, China) between December 2010 and February 2012. Secondary causes of renal syndrome (including allergic purpura nephritis, hepatitis B virus-associated glomerulonephritis, systemic lupus erythematosus nephritis, diabetic nephropathy, renal amyloidosis, myeloma kidney disease, lymphoma or solid tumours and kidney disease) were excluded during diagnosis. The present study was approved by the Ethics Committee of Hunan Provincial People's Hospital (Changsha, China), and informed consent was obtained from the patients.

Groups. The patients with renal syndrome were divided into two groups. In the first group (stable renal syndrome; $n=12$ ), urine protein tests became negative following treatment with sufficient amounts of a GC (prednisone) for 8-12 weeks. The disease remained stable during the gradual reduction of $\mathrm{GC}$ dosage. These 12 cases were in the long-term maintenance phase (minimum GC dose of $10 \mathrm{mg} /$ day). In the second group (renal syndrome recurrence group; $n=13$ ), urine protein tests became negative following treatment with sufficient amounts of GCs (prednisone) for 8-12 weeks. The 13 relapsed cases experienced a recurrence of renal syndrome when the GC dosage was gradually reduced or were in the long-term maintenance phase with existing infection factors; the conditions of these patients met the diagnostic criteria for nephrotic syndrome. No significant differences were observed in the age at the onset of disease, disease duration, hormone dosage, and the levels of nitrogen, creatinine and albumin in the blood urea between the two groups. All samples from the renal syndrome recurrence group were collected prior to antibiotic use.

A total of 10 postgraduates at the Hunan Provincial People's Hospital, First Affiliated Hospital of Hunan Normal University (Changsha, China) were selected as the normal control group. This group did not suffer from any infectious diseases and had not received any corticosteroids or immunosuppressants one week prior to the venous blood sampling. Thus, the experimental groups comprised 10 postgraduates as the normal control group (group A); 12 patients with controlled nephrotic syndrome as the stable renal syndrome group (group B); and 13 patients with recurrent nephrotic syndrome as the renal syndrome recurrence group (group C).

Furthermore, seven different in vitro treatment groups were used in the current experiments. For each experimental case, venous blood was collected and the peripheral blood mononuclear cells (PBMCs) were extracted. The PBMCs were added to Petri dishes prepared with nutrient solution for cultivation. A different additional stimulus was added to each group of Petri dishes to obtain the different treatment groups.
The details of the stimuli used were as follows: group 1 (blank group), no additional stimulus; group 2, TNF- $\alpha$; group 3, TNF- $\alpha+$ TNF- $\alpha$ monoclonal antibody; group 4 , methylprednisolone; group 5, TNF- $\alpha+$ methylprednisolone; group 6 , TNF- $\alpha+$ TNF- $\alpha$ monoclonal antibody + methylprednisolone and; group 7, TNF- $\alpha$ monoclonal antibody. The preparation and treatment of these groups is described in detail below.

Determination of the levels of TNF- $\alpha$. Heparin-anticoagulated fasting blood samples $(25 \mathrm{ml})$ were collected from all study participants at 8:00 a.m. and diluted with an equivalent amount of phosphate-buffered saline (PBS). Each sample mixture was slowly added to the surface of a Ficoll-Paque separation medium. The blood samples were centrifuged at $202 \mathrm{x} \mathrm{g}$ for $20 \mathrm{~min}$. The serum was collected and stored at $-80^{\circ} \mathrm{C}$ in a refrigerator until required. Finally, the TNF- $\alpha$ content in the serum was determined (R\&D Systems, Inc., Minneapolis, MN, USA) according to manufacturers' instructions.

PBMC isolation and culture. Heparin-anticoagulated fasting blood samples $(25 \mathrm{ml})$ were collected from all study participants at 8:00 a.m. and diluted with an equivalent amount of PBS. Each sample mixture was slowly added to the surface of a Ficoll-Paque separation medium. The blood samples were centrifuged at $202 \mathrm{x}$ g for $20 \mathrm{~min}$ (Xiangyi; Hunan Xiang Yi Laboratory Instrument Development Co., Ltd., Changsha, China). The mononuclear cell layers were subsequently removed with a Pasteur pipette, washed once with PBS and centrifuged at $250 \mathrm{x}$ g for $5 \mathrm{~min}$. The precipitated mononuclear cells were collected and resuspended in $10 \%$ fetal bovine serum (Hangzhou Sijiqing Biotechnology Co., Ltd, Hangzhou, China) and 1\% double anti-RPMI-1640 medium (Life Technologies, Shanghai, China) to a cell concentration of $5 \times 10^{4} / \mathrm{ml}$. Trypan blue staining was performed to determine cell viability (confirmed as $>95 \%$ ). A total of $3 \mathrm{ml}$ cell suspension was added to each well of seven 12 -well tissue culture wells. Following culturing for $6 \mathrm{~h}$ at $37^{\circ} \mathrm{C}$ under $5 \% \mathrm{CO}_{2}$, TNF- $\alpha$ (R\&D Systems China Co., Ltd, Shanghai, China), TNF- $\alpha+$ TNF- $\alpha$ monoclonal antibody (Epitomics, Burlingame, CA, USA), methylprednisolone, TNF- $\alpha+$ methylprednisolone, TNF- $\alpha+$ TNF- $\alpha$ monoclonal antibody + methylprednisolone, and TNF- $\alpha$ monoclonal antibody were added to the corresponding cell suspension groups. The group that did not receive any stimulation acted as the blank control. The blood samples of this group were further cultured for $24 \mathrm{~h}$ at $37^{\circ} \mathrm{C}$ under $5 \% \mathrm{CO}_{2}$. Cell viability was subsequently determined in all groups by Trypan blue staining (confirmed as $>95 \%$ ).

Protein expression. Cell culture supernatants were collected and stored at $-80^{\circ} \mathrm{C}$ in a refrigerator prior to use. The protein expression levels of GR, GR $\alpha$ and GR $\beta$ in the culture supernatants were determined using ELISA.

The optical density (OD) value of each well was measured using a microplate reader (Molecular Devices, Sunnyvale, CA USA) at $450 \mathrm{~nm}$. The corresponding concentrations in each well were calculated using a standard curve constructed by the CurveExpert 1.3 software (http://www.curveexpert.net. Accessed January 3, 2013) as a reference. The dilution factor was multiplied and the actual concentrations of the samples 
were obtained. A t-test was used when two samples demonstrated unequal variance.

mRNA detection. TRIzol ( $1 \mathrm{ml}$; Life Technologies) was added to the cell culture in each well and the wells were placed on ice for $5 \mathrm{~min}$. The cell cultures were subsequently transferred into $1.5 \mathrm{ml}$ Eppendorf polymerase chain reaction (PCR; EP) tubes following blowdown. Chloroform $(0.2 \mathrm{ml})$ was added to each tube. The tubes were agitated for $15 \mathrm{sec}$, placed on ice for $3 \mathrm{~min}$ and centrifuged at $4{ }^{\circ} \mathrm{C}$ and $15,984 \mathrm{x}$ g for $15 \mathrm{~min}$. The upper colourless liquid was transferred to a new EP tube and an equal volume of isopropanol (0.4-0.5 ml) was added. Subsequently, the solution was mixed, placed on ice for $30 \mathrm{~min}$ and centrifuged at $15,984 \mathrm{x}$ g for $10 \mathrm{~min}$ at $4^{\circ} \mathrm{C}$. The supernatant was removed, and ethanol $(750 \mu \mathrm{l})$ was added. Enzyme-free water $(250 \mu \mathrm{l})$ was added to the precipitate. The mixture was vortexed for $30 \mathrm{sec}$ and centrifuged at $6,243 \mathrm{x}$ g for $5 \mathrm{~min}$ at $4{ }^{\circ} \mathrm{C}$. The supernatant was removed and the precipitates in the tube were placed onto a clean workbench and dried by blast heating for 3-5 min. Diethylpyrocarbonate (DEPC) water (12 $\mu \mathrm{l}$ ) was added to dissolve the precipitates and the resulting solution was preserved at $-70^{\circ} \mathrm{C}$. To determine the RNA purity, the OD ratios of the total RNA at the optical densities of 260 and $280 \mathrm{~nm}$ were determined using a DU800 UV spectrophotometer (Beckman Coulter, Miami, FL, USA). The OD ratio was maintained at 1.8-2.0 and the RNA concentration was calculated.

Quantitative PCR ( $P$ PCR). The primers for qPCR were designed according to the principles of primer design. The National Center for Biotechnology Information (NCBI) GenBank database was searched and the primers were designed to amplify transcription products by PCR. Glyceraldehyde-3-phosphate dehydrogenase (GAPDH) was used as the internal reference for calibration. The primers were as follows: amplified fragment GR (114bp) upstream: 5'-CTGGATGACCAAATGACCCTA-3' and downstream: 5'-ATCAGGAGCAAAACACAGCAG-3'; amplified fragment GR $\alpha$ (172 bp) upstream: 5'-CCATTG TCAAGAGGGAAGGAAAC-3' and downstream: 5'-ATG ATTTCAGCTAACATCTCGGG-3'; amplified fragment GR $\beta$ (163 bp) upstream: 5'-CTG TCGCTTCTCAATCAGACTC-3' and downstream: 5'-CCCAGGTCATTTCCCATCACTT-3'; and for the amplified fragment GAPDH (256 bp) upstream: 5'-AGAAGGCTGGGGCTCATTTG-3' and downstream: 5'-AGGGGCCATCCACAGTCTTC-3'.

A 20- $\mu 1$ system was used for synthesis, which was performed in strict accordance with the manufacturers' instructions on the reverse transcriptase kit (Thermo Fisher Scientific, Beijing, China). The temperature was programmed as follows: $42^{\circ} \mathrm{C}$ for $60 \mathrm{~min}, 70^{\circ} \mathrm{C}$ for $5 \mathrm{~min}$ and infinite cycles at $4^{\circ} \mathrm{C}$. The cDNA product was stored at $-20^{\circ} \mathrm{C}$ until required.

A $15-\mu 1$ system comprising $1.5 \mu \mathrm{l}$ cDNA sample, $0.6 \mu \mathrm{l}$ target gene primers, 7.5 $\mu 1 \mathrm{SYBR}-\mathrm{Green}$ and 5.4 $\mu \mathrm{l}$ enzyme-free water were added to a 96 -well plate. The mixtures were centrifuged at $999 \times \mathrm{g}$ for $5 \mathrm{~min}$ at $4^{\circ} \mathrm{C}$ and placed into a $7300 \mathrm{PCR}$ instrument (Life Technologies) for analysis.

Western blot analysis. Western blot analysis was used to isolate and electrically transfer $40 \mu \mathrm{g}$ total cellular protein from a $6 \%$ denaturing polyacrylamide gel to a polyvinylidene difluoride (PVDF) membrane film. The film was sealed with bovine serum albumin and reacted with rabbit anti-human GR (1:1,000 dilution), GR $\alpha$ (1:1,000 dilution) and GR $\beta$ (1:500 dilution) antibodies (R\&D Systems China Co., Ltd) for $2 \mathrm{~h}$. Subsequently, the mixtures were reacted with horseradish peroxidase-labelled anti-rabbit immunoglobulin $\mathrm{G}(\mathrm{IgG}$; Thermo Scientific Pierce Protein Biology Products, Rockford, IL, USA) for 45 min until diaminobenzidine (DAB)-coloured bands appeared. OD scan results for the positive bands revealed that GR, GR $\alpha$ and GR $\beta$ proteins were specifically expressed in the PBMCs at molecular weights of 94,300, 94,000 and 90,000 kDa, respectively.

Statistical analysis. Cycle threshold (CT) values were obtained using the 7300 system SDS software installed on the instrument. GAPDH was used as the internal reference and was described using the $2-{ }^{\Delta \Delta C T}$ ratio values, determined using SPSS software, version 17.0 (SPSS, Inc., Chicago, IL, USA) in the stimulation and blank groups. The experimental data are expressed as the mean \pm standard deviation and were analysed with SPSS 17.0 statistical software. The differences between multiple samples were analysed by ANOVA. Comparisons between two samples were performed using the Student' t-test. A t-test was used when two samples demonstrated unequal variance.

\section{Results}

Determination of the levels of TNF- $\alpha$. The serum levels of TNF- $\alpha$ in the normal, stable renal syndrome and renal syndrome recurrence groups were $6.48 \pm 1.34,7.06 \pm 1.89$ and $20.35 \pm 1.79 \mathrm{pg} / \mathrm{ml}$, respectively. No significant differences were observed in the serum levels of TNF- $\alpha$ between the stable renal syndrome and normal groups. However, the serum levels of TNF- $\alpha$ in the renal syndrome recurrence group were significantly higher compared with those in the normal and stable renal syndrome groups $(\mathrm{P}<0.01)$.

Expression levels of $G R$ proteins and $m R N A$. Prior to intervention, the expression levels of the GR protein and mRNA in the PBMC culture supernatants and PBMCs of the stable renal syndrome and renal syndrome recurrence groups were not significantly different from those in the normal group. Furthermore, in the stable renal syndrome group, the expression levels of the GR $\alpha$ and GR $\beta$ proteins and mRNA in the PBMC culture supernatants and PBMCs were not significantly different from those in the normal group. The GR $\alpha$ protein and mRNA expression levels in the PBMC culture supernatants and PBMCs in the renal syndrome recurrence group were lower compared with those in the normal control and stable renal syndrome groups $(\mathrm{P}<0.05$; prior to intervention data in Tables I-III). Furthermore, GR $\beta$ mRNA expression levels in the renal syndrome recurrence group were significantly higher compared with those in the normal control and stable renal syndrome groups $(\mathrm{P}<0.05$; prior to intervention data in Tables I-III).

$G R$ proteins and $m R N A$ expression following treatment with $T N F-\alpha$. Following intervention via TNF- $\alpha$ stimulation, the expression levels of the GR and GR $\beta$ proteins and mRNA in 
政

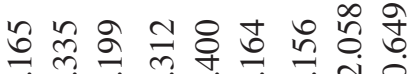

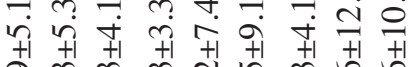

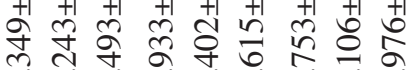

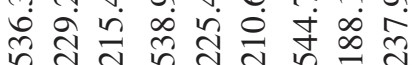

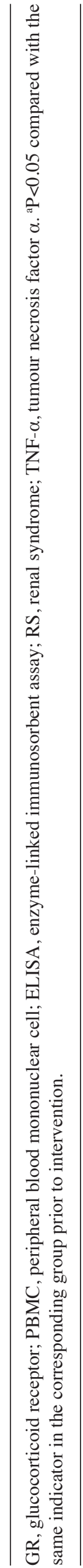
范 \&

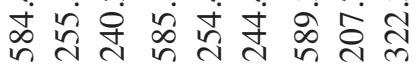

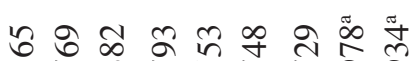

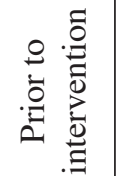

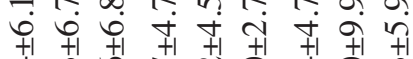

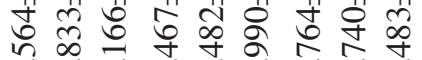

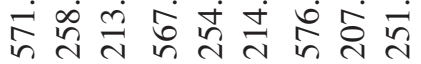

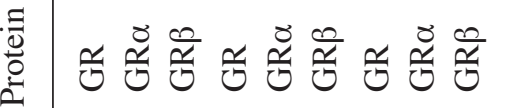
i

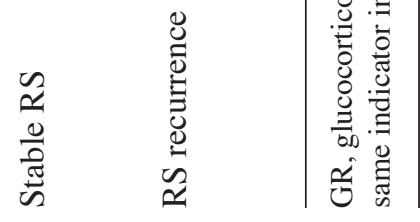

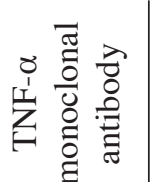

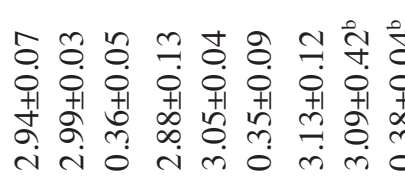

음 중 至 焉 +

원 흥

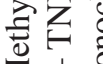
$\sum+$ g

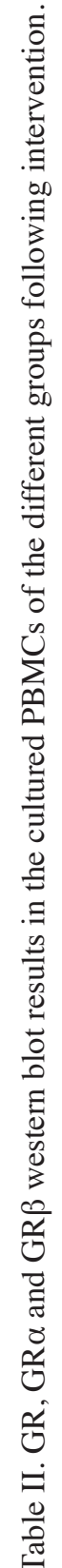

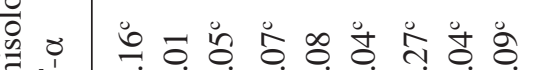

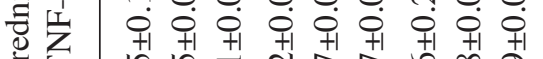

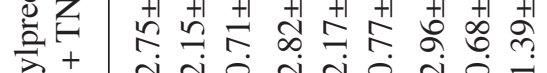
产+ $\Sigma$

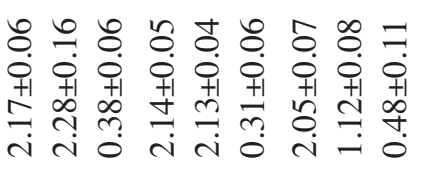
용 a 용 8 응

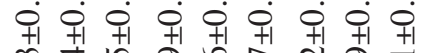

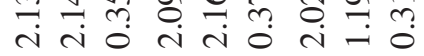

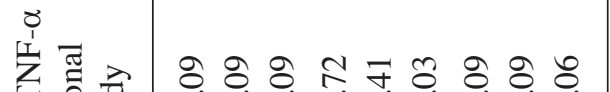

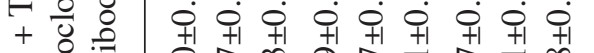
诘言

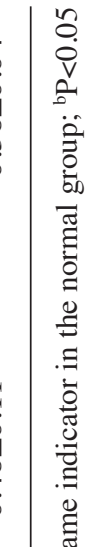
导 量 言 s. v 哣. 远 . 诺

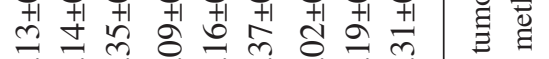
i임 宸. 范 突号 苛

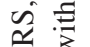

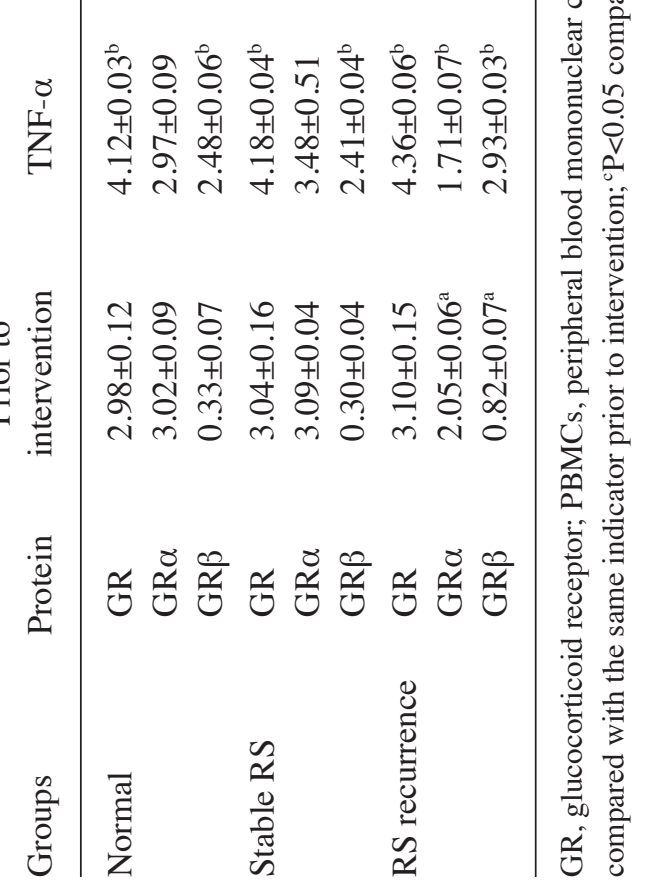


Table III. 2- ${ }^{\triangle A C T}$ values of GR, GR $\alpha$ and GR $\beta$ mRNA in the PBMC culture supernatants of the different groups detected by quantitative poymerase chain reaction following intervention.

\begin{tabular}{lccc}
\hline & \multicolumn{3}{c}{ Group } \\
\cline { 2 - 4 } Items & Normal & Stable RS & RS recurrence \\
\hline GR & 1 & $1.044 \pm 0.108$ & $0.964 \pm 0.087$ \\
GR $\alpha$ & 1 & $0.979 \pm 0.088$ & $0.778 \pm 0.045^{\mathrm{a}}$ \\
GR $\beta$ & 1 & $1.093 \pm 0.145$ & $1.394 \pm 0.101^{\mathrm{a}}$ \\
\hline
\end{tabular}

GR, glucocorticoid receptor; PBMC, peripheral blood mononuclear cell; RS, renal syndrome; TNF- $\alpha$, tumour necrosis factor $\alpha$; CT, cycle threshold. ${ }^{a} \mathrm{P}<0.05$ compared with the same indicator in the normal group.

the PBMC culture supernatants and PBMCs of the normal, stable renal syndrome and renal syndrome recurrence groups were higher compared with those of the levels of the blank control in each group $(\mathrm{P}<0.05$; Tables I, II and IV). Following treatment with TNF- $\alpha$ and TNF- $\alpha$ monoclonal antibodies, the expression levels of the GR and GR $\beta$ proteins and mRNA in the PBMC culture supernatants and PBMCs of the normal, stable renal syndrome and renal syndrome recurrence groups were lower compared with the levels following TNF- $\alpha$ stimulation in the respective groups $(\mathrm{P}<0.05$; Tables I, II and IV) and were not significantly different from those of the blank control group. Following intervention by TNF- $\alpha$ stimulation, the GR $\alpha$ protein levels in the PBMC culture supernatants and PBMCs and GR $\alpha$ mRNA expression levels in the PBMCs of the renal syndrome recurrence group were lower than those in the blank control $(\mathrm{P}<0.05$; Tables I, II and IV). In addition, following treatment of the renal syndrome recurrence group with TNF- $\alpha$ and TNF- $\alpha$ monoclonal antibody, the GR $\alpha$ protein and mRNA expression levels were higher than those following TNF- $\alpha$ stimulation ( $\mathrm{P}<0.05$; Tables I, II and IV) and were not significantly different from those of the control.

Expression levels of GR proteins and mRNA following TNF- $\alpha$, monoclonal antibody and methylprednisolone stimulation. Following intervention through methylprednisolone $+\mathrm{TNF}-\alpha$ stimulation, the expression levels of the GR and GR $\beta$ proteins in the PBMC culture supernatants and PBMCs and of GR and GR $\beta$ mRNA in the PBMCs of the normal, stable renal syndrome and renal syndrome recurrence groups were higher compared with those of the corresponding group following methylprednisolone treatment $(\mathrm{P}<0.05$; Tables I, II and IV). Following treatment with methylprednisolone + TNF- $\alpha+$ TNF- $\alpha$ monoclonal antibody, the expression levels of the GR and GR $\beta$ proteins in the PBMC culture supernatants and PBMCs and the expression levels of GR and GR $\beta$ mRNA in the PBMCs of the normal, stable renal syndrome and renal syndrome recurrence groups were lower compared with those in the corresponding group following treatment with methylprednisolone + TNF- $\alpha$ $(\mathrm{P}<0.05$; Tables I, II and IV) and revealed no significant differences from those following methylprednisolone treatment. Following methylprednisolone + TNF- $\alpha$ intervention, the levels of GR $\alpha$ protein expression in the PBMC culture

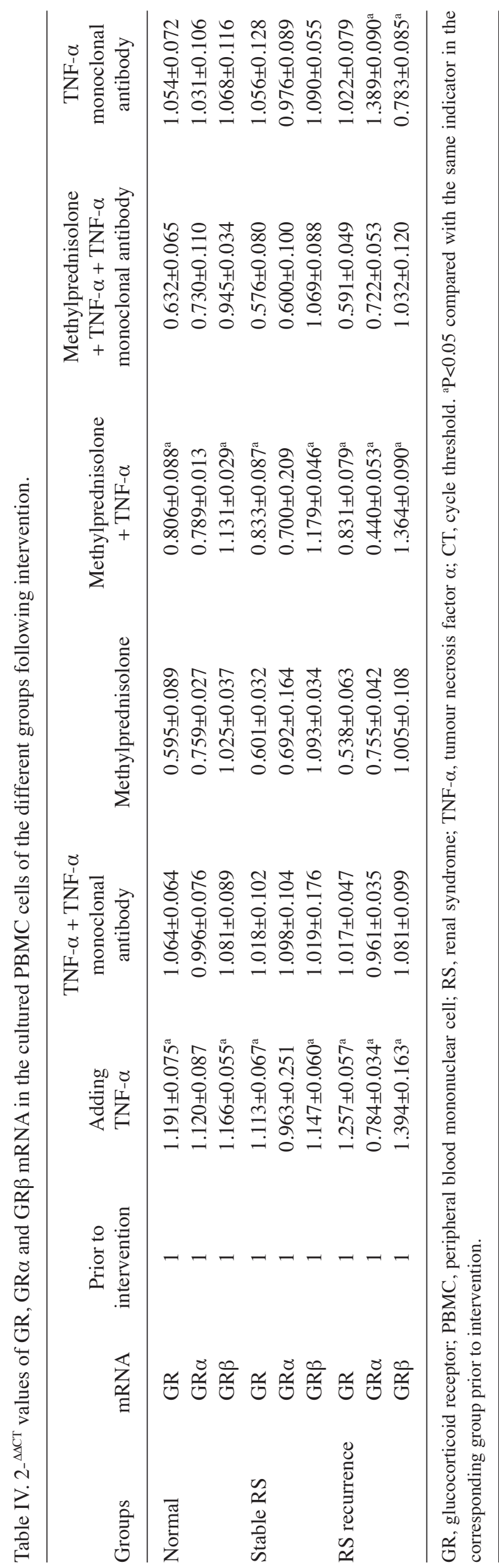


supernatants and PBMCs and of GR $\alpha$ mRNA expression in the PBMCs of the renal syndrome recurrence group were lower compared with those of the control group $(\mathrm{P}<0.05$; Tables I, II and IV). Furthermore, the GR $\alpha$ protein and mRNA expression levels following methylprednisolone $+\mathrm{TNF}-\alpha+\mathrm{TNF}-\alpha$ monoclonal antibody stimulation were higher than those following methylprednisolone $+\mathrm{TNF}-\alpha$ stimulation in the renal syndrome recurrence group $(\mathrm{P}<0.05$; Tables I, II and IV) and revealed no significant difference from those following methylprednisolone treatment.

Expression levels of GR proteins and $m R N A$ following $T N F-\alpha$ monoclonal antibody treatment. Following the addition of the TNF- $\alpha$ monoclonal antibody, no significant differences were observed in GR protein expression in the PBMC culture supernatants and PBMCs and in the GR mRNA expression in the PBMCs of the normal, stable renal syndrome and relapsed renal syndrome groups. The GR $\alpha$ protein and mRNA expression of the renal syndrome recurrence group following treatment with the TNF- $\alpha$ monoclonal antibody was significantly higher compared with that of the blank control $(\mathrm{P}<0.05$; Tables I, II and IV). No significant difference in GR $\alpha$ protein or mRNA expression was observed between the stable renal syndrome and the blank control groups. GR $\beta$ protein expression in the PBMC culture supernatants and PBMCs and GR $\beta$ mRNA expression in the PBMCs of the renal syndrome recurrence group following TNF- $\alpha$ monoclonal antibody treatment were lower compared with those of the blank control ( $\mathrm{P}<0.05$; Tables I, II and IV); no significant differences in GR $\beta$ expression levels were observed between the stable renal syndrome and normal groups.

\section{Discussion}

The mechanism underlying nephrotic syndrome recurrence remains unclear. Certain researchers believe that this syndrome is associated with GR expression and isoform changes (14-16). The current study revealed that GR $\alpha$ protein and mRNA expression levels in the PBMCs of the renal syndrome recurrence group were lower compared with those of the normal control and stable renal syndrome groups. However, the expression levels of GR and GR $\beta$ mRNA and protein in the PBMCs of the relapsed renal syndrome group were higher compared with those in the normal control group. These results indicate that nephrotic syndrome recurrence is associated with GR expression levels, reductions in GR $\alpha$ expression and overexpression of GR $\beta$. Renal syndrome is caused by various factors, including infections, which may trigger the recurrence of renal syndrome by inducing the GR to alter the efficiency of GCs.

Immune inflammatory mediators and transcription factors are vital to the regulation of GR-mediated GC efficacy. Various inflammatory response stimuli are able to reduce or even inhibit the binding of GC to target cells. This phenomenon is the clinical reason for recurrent nephrotic syndrome as well as for the recurrence of other inflammatory reactions. Infection is the most common cause of relapse. Bacterial cell components, including endotoxin lipopolysaccharide (LPS) and lipoteichoic acid (LTA), are able to activate the monocyte/macrophage intracellular signal transduction pathways and induce the transcription and overexpression of the genes encoding various inflammatory media, including TNF- $\alpha$. During the early stages of inflammation, TNF- $\alpha$ may be used as a key medium to induce the expression of downstream factors, including interleukin (IL)-1, IL-2, IL-4, IL- 8 and $\lambda$-interferon (IFN), and th $\gamma$ us initiate a cascade reaction, which further promotes the recurrence of nephrotic syndrome by affecting GC response. A number of studies have revealed that inflammatory response factors are able to reduce or even inhibit GC activity by affecting the GR $\alpha / G R \beta$ ratio and GR function (17-21). TNF- $\alpha$ is an important pleiotropic cytokine with a wide range of biological functions. The GC-GR complex may directly or indirectly inhibit the production and release of TNF- $\alpha$ and other inflammatory cytokines. TNF- $\alpha$ may also induce the reduction or even inhibition of GC activity and thus directly affect the GR (22).

The current study revealed that the serum levels of TNF- $\alpha$ in patients with nephrotic syndrome recurrence were significantly higher compared with those in the normal and stable renal syndrome groups. This suggests that increased serum levels of TNF- $\alpha$ are associated with the recurrence of nephrotic syndrome. Following stimulation with TNF- $\alpha$ alone or with TNF- $\alpha$ combined with methylprednisolone, the protein expression levels of GR and GR $\beta$ in the culture supernatants and PBMCs and the mRNA expression levels of GR and GR $\beta$ in the PBMCs in the normal, stable renal syndrome and renal syndrome recurrence groups were higher compared with those of the respective blank control. However, GR $\alpha$ protein expression levels in the culture supernatants and PBMCs and GR $\alpha$ mRNA expression levels in the PBMCs of the renal syndrome recurrence group following stimulation with TNF- $\alpha$ were lower compared with those of the blank control. Following the addition of TNF- $\alpha$ monoclonal antibodies to antagonise TNF- $\alpha$ stimulation, the expression levels of GR and GR $\beta$ proteins in the culture supernatants and PBMCs and the expression levels of GR and GR $\beta$ mRNA in the PBMCs of the normal, stable renal syndrome and renal syndrome recurrence groups were lower compared with those following treatment with TNF- $\alpha$-alone or TNF- $\alpha+$ methylprednisolone. No significant differences were observed from the blank control in each group.

Following the addition of TNF- $\alpha$ monoclonal antibody, the levels of GR $\alpha$ protein and mRNA expression in the PBMCs of the renal syndrome recurrence group were higher compared with those of the control group. However, the expression levels of GR and GR $\beta$ mRNA in this group were lower compared with those in the control. Following the addition of TNF- $\alpha$ monoclonal antibodies to the stable renal syndrome group, no significant differences were observed in the expression levels of GR and GR $\beta$ proteins and mRNA between the stable renal syndrome and control groups. These results indicate that TNF- $\alpha$ may be involved in nephrotic syndrome recurrence by altering the levels and proportions of the GR subtypes. TNF- $\alpha$ monoclonal antibodies exhibited an antagonistic effect on TNF- $\alpha$ activity that was associated with changes in the proportion of the GR subtypes. Therefore, treatments involving TNF- $\alpha$ targeting may disrupt the inflammatory response and amplification and thus alleviate or suppress the inflammatory factors that affect GR. This approach may effectively improve GC reactivity in patients with renal syndrome. 


\section{References}

1. Zhang HW, Wang F and Ding J: Updates on the molecular genetics of congenital nephrotic syndrome. Zhonghua Er Ke Za Zhi 49: 425-427, 2011 (In Chinese)

2. Stahl RA, Hoxha E and Helmchen U: Membranous glomerulonephritis: better therapy with autoantibody monitoring? Dtsch Med Wochenschr 136: 1733-1737, 2011 (In German).

3. Bruneau S, and Dantal J: New insights into the pathophysiology of idiopathic nephrotic syndrome. Clin Immunol 133: 13-21, 2009.

4. Bomback AS and Radhakrishnan J: Treatment of nephrotic syndrome with adrenocorticotropic hormone (ACTH). Discov Med 12: 91-96, 2011.

5. Saito T: Treatment and prognosis of idiopathic membranous nephropathy in guidelines for nephrotic syndrome. Nihon Jinzo Gakkai Shi 53: 708-712, 2011 (In Japanese).

6. Guess A, Agrawal S, Wei CC, Ransom RF, Benndorf R and Smoyer WE: Dose- and time-dependent glucocorticoid receptor signaling in podocytes. Am J Physiol Renal Physiol 299: F845-F853, 2010.

7. Wang L, Li Q, Wang LJ and Li X: Level of Th17 cell and CD4(+); CD25(+); Foxp3(+); regulatory $\mathrm{T}$ cell in peripheral blood mononuclear cells of primary nephrotic syndrome in children. Xi Bao Yu Fen Zi Mian Yi Xue Za Zhi 26: 783-786, 2010 (In Chinese).

8. Farid FA, Mohammed AA, Afifi HM and Beltagi RS. Tissue factor pathway inhibitor in paediatric patients with nephrotic syndrome. SAJCH 5: 107-111, 2011.

9. Tan Y, Yang D, Fan J and Chen Y: Elevated levels of immunoglobulin E may indicate steroid resistance or relapse in adult primary nephrotic syndrome, especially in minimal change nephrotic syndrome. J Int Med Res 39: 2307-2313, 2011.

10. Audard V, Pawlak A, Candelier M, Lang P and Sahali D: Upregulation of nuclear factor-related kappa B suggests a disorder of transcriptional regulation in minimal change nephrotic syndrome. PLoS One 7: e30523, 2012.

11. Caridi G, Trivelli A, Sanna-Cherchi S, Perfumo F, Ghiggeri GM: Familial forms of nephrotic syndrome. Pediatr Nephrol 25: 241-252, 2010

12. Elie V, Fakhoury M, Deschênes G and Jacqz-Aigrain E: Physiopathology of idiopathic nephrotic syndrome: lessons from glucocorticoids and epigenetic perspectives. Pediatr Nephrol 27: 1249-1256, 2012.
13. Segal PE and Choi MJ: Recent advances and prognosis in idiopathic membranous nephropathy. Adv Chronic Kidney Dis 19: 114-119, 2012.

14. Shalaby SA, El Idrissy HM, Safar RA and Hussein ST: Glucocorticoid receptors and the pattern of steroid response in idiopathic nephrotic syndrome. Arab J Nephrol Transplant 5: 13-17, 2012

15. Szilagyi K, Podracka L, Franke NE, Mojzis J and Mirossay L: A new link between steroid resistance, glucocorticoid receptor and nuclear factor kappa B p65 in idiopathic nephrotic syndrome. Neuro Endocrinol Lett 30: 629-636, 2009.

16. Chen P, Jiang T, Ouyang J and Cui Y: Glucocorticoid receptor auto-upregulation and its relation with glucocorticoid sensitivity in idiopathic nephrotic syndrome. Int Urol Nephrol 43: 167-174, 2011.

17. Pace TW, Hu F, Miller AH: Cytokine-effects on glucocorticoid receptor function: relevance to glucocorticoid resistance and the pathophysiology and treatment of major depression. Brain Behav Immun 21: 9-19, 2007.

18. Wang X, Wu H, Lakdawala VS, Hu F, Hanson ND and Miller AH: Inhibition of Jun N-terminal kinase (JNK) enhances glucocorticoid receptor-mediated function in mouse hippocampal HT22 cells. Neuropsychopharmacology 30: 242-249, 2005.

19. Wang X, Wu H and Miller AH: Interleukin 1alpha (IL-1alpha) induced activation of p38 mitogen-activated protein kinase inhibits glucocorticoid receptor function. Mol Psychiatry 9: $65-75,2004$.

20. Rogatsky I and Ivashkiv LB: Glucocorticoid modulation of cytokine signaling. Tissue Antigens 68: 1-12, 2006.

21. Biola A, Lefebvre P, Perrin-Wolff M, Sturm M, Bertoglio J, Pallardy M: Interleukin-2 inhibits glucocorticoid receptor transcriptional activity through a mechanism involving STAT5 (signal transducer and activator of transcription 5) but not AP-1. Mol Endocrinol 15: 1062-1076, 2001.

22. Kam JC, Szefler SJ, Surs W, Sher ER and Leung DY: Combination IL-2 and IL-4 reduces glucocorticoid receptor-binding affinity and $\mathrm{T}$ cell response to glucocorticoids. J Immunol 151: 3460-3466, 1993. 